\title{
GLEASON PARTS AND CERTAIN COUNTEREXAMPLES IN THE BIG DISC CONTEXT
}

\author{
J. P. MILASZEWICZ
}

ABSTRACT. In the first part of this paper, Lemmas (1.1) and (1.4) provide a generalization of the fact that no Cauchy measures exist in the big disc. In the second part we show that this fact implies the existence of the $\mathrm{H}$. Bohr and J. Favard counterexamples concerning harmonic and analytic almost periodic functions.

Introduction. It is well known and easy to prove that in the big disc there are many Gleason parts out of the Šilov boundary of the big disc algebra. Our aim in this note is to show how this fact implies (i) there are harmonic almost periodic functions in a half-plane such that the ir harmonic conjugates are not almost periodic in any strictly contained half-plane (see [6, Chapitre III, \$47]), and (ii) there are analytic almost periodic functions in a strip with no Laurent decomposition (see [5, p. 278]).

1. Gleason parts and uniform approximation. We say that $A$ is a uniform a lgebra over the compact space $X$ if (i) $A \subset C(X)$ and it is closed in the uniform convergence topology, (ii) $A$ separates the points of $X$ and contains the constant functions. We denote the spectrum of $A$ by $\operatorname{Sp}(A)$. If $x, y \in \mathrm{Sp}(A),\|x-y\|$ will be the norm of $x-y$ as an element of the dual space of $A$. If $x \in \mathrm{Sp}(A)$ we write $A_{x}=\{f \in A: \hat{f}(x)=0\}, \hat{f}$ being the Gelfand transform of $f$. If $m \in M(X)=$ \{bounded measures on $X\}, \bar{m}$ is the measure defined by $\bar{m}(f)=\overline{m(\bar{f})}$ (the conjugate measure). If $x \in \mathrm{Sp}(A), K_{x}$ is the set of representing measures for $x$. If $m \in M(X)$ we write $m \perp A$ when $m$ is orthogonal to $A$.

(1.1) Lemma. Let $A$ be a uniform algebra over $X$ and $x, y \in \mathrm{Sp}(A)$. The following are equivalent:

(i) $\|x-y\|=2$;

(ii) $1 \in$ Closure of $\left(A_{x}+\bar{A}_{y}\right)=\mathrm{Cl}\left(A_{x}+\bar{A}_{y}\right)\left(\bar{A}_{y}=\left\{\bar{f}: f \in A_{y}\right\}\right)$.

Proof. (ii) $\Rightarrow$ (i). Let $f_{n} \in A_{x}$ and $g_{n} \in A_{y}$ be such that $f_{n}+g_{n}$ con-

Received by the editors March 8, 1973 and, in revised form, August 29, 1973. AMS (MOS) subject classifications (1970). Primary 46J10; Secondary 42A84. 
verges uniformly to 1 . Suppose that $\|x-y\|<2$; then there exist $m_{x}$ and $m_{y}$, representing measures of $x$ and $y$ respectively and $c>0$ such that (a) $c^{-1} m_{y} \leq m_{x} \leq c m_{y}$ (see [4]). As $\left(1-f_{n}\right) \perp \bar{g}_{n}$ in $L^{2}\left(m_{y}\right)$ we have

$$
\int\left|1-f_{n}-\bar{g}_{n}\right|^{2} d m_{y}=\int\left|1-f_{n}\right|^{2} d m_{y}+\int\left|g_{n}\right|^{2} d m_{y}=k_{n} .
$$

$k_{n}$ converges to zero as $n$ tends to infinity; hence, $f_{n}$ and $g_{n}$ converge in $L^{2}\left(m_{y}\right)$ to 1 and 0 respectively. This implies, because of (a), that the same occurs in $L^{2}\left(m_{x}\right)$. On the other hand, by simple conjugation, we can obtain that $g_{n}$ converges to 1 in $L^{2}\left(m_{x}\right)$. This contradiction implies (i).

(i) $\Rightarrow$ (ii). Suppose $n \perp\left(A_{x}+\bar{A}_{y}\right)$; we want to show that $n \perp 1$. If $n=$ $n_{x}+n_{s}^{\prime}$ is the generalized Lebesgue decomposition of $n$ with respect to $K_{x}$ (see [7, Chapter II, Corollary 7.5]), the abstract F. and M. Riesz theorem (see [7, Chapter II, Corollary 7.9]) gives us that $n_{x} \perp A_{x}$ and $n_{s}^{\prime} \perp A$; hence

$$
n(1)=n(X)=\int d n=n_{x}(1) .
$$

We recall that $n_{x}$ is absolutely continuous with respect to some $m_{x}$ in $K_{x}$ and that there exists a Borel set $E_{x}$ such that $m_{x}\left(E_{x}\right)=0$ whenever $m_{x} \epsilon$ $K_{x}$ and $\left\|n_{s}^{\prime}\right\|\left(X-E_{x}\right)=0$. Because of (i), $E_{x}$ can be chosen in such a way that $m_{y}\left(X-E_{x}\right)=0$ for every $m_{y}$ in $K_{y}$. (See [7, Chapter VI, Theorem 2.2].) Consider $n_{s}^{\prime}$ and $K_{y}$ and proceed in the same way as with $n$; i.e., $n_{s}^{\prime}=$ $n_{y}+n_{s}$ is the Lebesgue decomposition of $n_{s}^{\prime}$ with respect to $K_{y}, E_{y} \subset E_{x}$ is such that $\left\|n_{s}\right\|\left(X-E_{y}\right)=0, m_{y}\left(E_{y}\right)=0$ whenever $m_{y} \in K_{y}$ and $n_{s} \perp A$. Now we have

$$
0=n_{s}^{\prime}(1)=n_{y}(1)=n_{s}(1) .
$$

Consider $\bar{n}=\bar{n}_{y}+n^{\prime}$ (here $n^{\prime}=\bar{n}_{x}+\bar{n}_{s}$ ); $n^{\prime}$ is concentrated in $E_{y}^{\prime}=$ $E_{y} \cup\left(X-E_{x}\right)$ and $m_{y}\left(E_{y}^{\prime}\right)=0$ whenever $m_{y} \in K_{y}$; we have also that $\bar{n}_{y}$ is absolutely continuous $w$ ith respect to some $m_{y} \in K_{y}$. Thus, $\bar{n}=\bar{n}_{y}+n^{\prime}$ is the Lebesgue decomposition of $\bar{n}$ with respect to $K_{y}$; as $\bar{n} \perp A_{y}$ we get that $n^{\prime} 1 A$ (F. and M. Riesz theorem). This remark and (c) imply that $n_{x}(1)$ $=0$; going back to $(\mathrm{b})$ we reach the conclusion.

(1.2) Corollary. Let $A$ be a Dirichlet algebra; $x, y \in \mathrm{Sp}(A)$. Then $\|x-y\|=2$ iff $A_{x}+\bar{A}_{y}$ is dense in $C(X)$.

In all that follows we shall use the notations concerning the big disc as in [10]; in particular, $\Gamma$ will indicate the dual group of any dense subgroup 
$G$ of the real numbers, when $G$ is endowed with the discrete topology.

(1.3) Corollary. Let $A$ be the big disc albegra and $0<r<1$; there does not exist $m_{r}^{\prime} \in M(\Gamma)$ such that

$$
\hat{m}_{r}^{\prime}(x)=\left\{\begin{array}{ll}
|x| & \forall x \leq 0, \\
0 & \forall x>0,
\end{array} \quad x \in G .\right.
$$

Proof. Suppose that such an $m_{r}^{\prime}$ exists; then, $m_{r}^{\prime} \perp \bar{A}_{0}$ and $m_{r}^{\prime} \perp A_{r}$. Because of (1.1) we must have that $m_{r}^{\prime}(1)=0$ which is a contradiction.

Remark. Were $G$ the set of rational integers, measures like $m_{r}^{\prime}$ in (1.3) would exist; explicitly, $m_{r}^{\prime}$ is the Cauchy kernel $c_{r}(\theta)=(2 \pi)^{-1}\left(1-r e^{-i \theta}\right)^{-1}$, $(\theta \in[0,2 \pi))$.

Corollary (1.3) is a particular case of Theorem (6.4) in [10] and it will be the sole fact used in the second part of this note. As the final result of this section we give a generalization of Theorem (6.4) in [10].

(1.4) Lemma. Let $A$ be a Dirichlet algebra, $x \in \mathrm{Sp}(A)$ and $m_{x}$ its representing measure. If $n \perp A_{x}$ and there exists a real measure $m \in M(X)$, $m$ mutually singular with $m_{x}$, such that $m(\bar{f})=n(\bar{f})$ whenever $f \in A$, then $n(1)=0$.

Proof. It is not difficult to check that

$$
(n+\bar{n})(g)=\left(m+m(1) m_{x}\right)(g) \quad \forall g \in \operatorname{Re}(A)
$$

so that $n+\bar{n}=m+m(1) m_{x}$.

Let $n=h m_{x}+n_{s}$ be the Lebesgue decomposition of $n$ with respect to $m_{x}$; hence $n+\bar{n}=2 \operatorname{Re} h m_{x}+\left(n_{s}+\bar{n}_{s}\right)$ is the Lebesgue decomposition of $n+\bar{n}$ with respect to $m_{x}$. As $m$ is singular with respect to $m_{x}$ we get that $m=n_{s}+\bar{n}_{s}$; on the other hand, because of the F. and M. Riesz theorem (see [7, Chapter II, Corollary 7.9]), we have that $n_{s} \perp A$ so that $0=n_{s}(1)+\bar{n}_{s}(1)$ $=m(1)=n(1)$.

2. Some consequences in the big disc. In $L^{2}(\Gamma)$ we have the Hilbert conjugation operator defined by $H(f)=\Sigma \hat{f}(x) \operatorname{sgn}(x) \hat{x}$. (Here $\hat{x}$ is the character associated to $\left.x_{\bullet}\right) H$ is an isometry because of Plancherel's theorem. If $0<r<1, m_{r}$ will denote the representing measure of $r$ (see [10]); recall that $\hat{m}_{r}(x)=r|x| \forall x \in G$. Fixing $r$ we can define a new operator on $L^{2}(\Gamma)$ by convolution with $m_{r}$, namely $S(f)=H(f) * m_{r}$. If we were dealing with 
the little disc, it would be easy to see that $S(f)$ is a continuous function in the unit circle; we shall show that it is not the case in our context, even when $f$ is continuous, i.e., there exist continuous functions $f$ in $\Gamma$ such that $S(f)$ is not. Consider $L^{1}(\Gamma)$ and define $i: L^{1}(\Gamma) \rightarrow L^{\infty}(\Gamma)^{*}$ by $i(f)(g)=$ $\int f g d m \forall g \in L^{\infty}$ ( $m$ is normalized Haar measure). Define $i_{1}: L^{1}(\Gamma) \rightarrow M(\Gamma)$ analogously. It is a well-known fact that $i$ and $i_{1}$, preserve the norms.

(2.1) Theorem. There exists $f \in C(\Gamma)$ such that $S(f) \notin L^{\infty}(\Gamma)$.

Proof. Suppose that $S(f) \in L^{\infty}(\Gamma) \forall f \in C(\Gamma)$; we have then a new operator $T$ defined on $C(\Gamma)$ with range in $L^{\infty}(\Gamma)$. Let us prove that $T$ is continuous when $C(\Gamma)$ and $L^{\infty}(\Gamma)$ have the essential supremum norm; for this we apply the closed graph theorem. Let $f_{n}$ converge uniformly to $f\left(f_{n}, f \in\right.$ $C(\Gamma)$ ) and $T\left(f_{n}\right)$ converge to $g$ in $L^{\infty}(\Gamma)$; clearly, we have that $f_{n}$ converges to $f$ in $L^{2}(\Gamma)$ and $T\left(f_{n}\right)=S\left(f_{n}\right)$ converges to $S(f)=T(f)$ (S being continuous on $\left.L^{2}(\Gamma)\right)$. Hence $g=S(f)=T(f)$.

Consider now the dual mapping $T^{*}$ between the dual spaces; it is a simple verification that $T^{*}(i(f))=i_{1}(T(f)) \forall f \in C(\Gamma)$. This implies that the restriction of $T^{*}$ to $i\left(L^{1}(\Gamma)\right)$ has its image in $i_{1}\left(L^{1}(\Gamma)\right)$; as $i$ and $i_{1}$ are isometries we have then a bounded operat or $T_{i}: L^{1}(\Gamma) \rightarrow L^{1}(\Gamma)$.

As $T_{i}(f * g)=\left(T_{i}(f)\right) * g$ whenever $f$ and $g$ are in $C(\Gamma)$, the same holds when $f$ and $g$ are in $L^{1}(\Gamma)$. Such an operator is necessarily the convolution with a measure $\tilde{n}$. But then

$$
\hat{\tilde{n}}(x)=(\tilde{n} * \hat{x})(0)=\left(T_{i}(\hat{x})\right)(0)=\left\{\begin{array}{ll}
r^{x} \hat{x}(0)=r^{x} & \text { if } x \geq 0, \\
-r|x| & \text { if } x<0,
\end{array} \quad x \in G,\right.
$$

so that the measure $m_{r}^{\prime}=2^{-1}\left(m_{r}+\overline{\tilde{n}}\right)$ produces a contradiction with (1.3).

In all that follows we use the definitions and results concerning almost periodic (a.p.) functions as stated in [3].

In [2] the big disc algebra associated to $G$ is identified isometrically with the algebra of continuous bounded a.p. functions in $P=\{\operatorname{Re} w>0\}$, analytic in $\{\operatorname{Re} w>0\}$ and whose exponents lie in $G$; in the same way it is possible to identify $C(\Gamma)$ with the space of a.p. functions in the imaginary axis or with the space of continuous bounded a.p. functions in $P$, harmonic in its interior; it is not difficult to prove that if $u$ is a harmonic bounded function in a vertical strip and $v$ is its harmonic conjugate, then if $v$ is bounded on one vertical line contained in the strip, $v$ must be bounded on every other vertical line of the strip. With these remarks the meaning of 
(2.1) is (i) in the introduction. Explicitly, there exists a continuous bounded a.p. function in $P$, harmonic in $\{\operatorname{Re} w>0\}$ such that its harmonic conjugate is not a.p. (not even bounded) in any proper half-plane $\{\operatorname{Re} w \geq k>0\}$.

Our aim now is to prove (ii) in the introduction. Let $B$ be the algebra of continuous bounded a.p. functions in $\{0 \leq \operatorname{Re} w \leq 1\}$, analytic in the interior of the strip. We call $A_{1}$ the algebra of continuous bounded a.p. functions in $\{\operatorname{Re} w \geq 0\}$, a nalyt ic in $\{\operatorname{Re} w>0\} ; A_{1}^{0}$ is the algebra of continuous bounded a.p. functions in $\{\operatorname{Re} w \leq 1\}$, a nalytic in its interior and vanishing at infinity.

The algebra $B$ can be identified with the algebra $B_{G}$ in $[12$, Example 5] (here $G=R$ ) and its Šilov boundary is $\Gamma \cup e^{-1} \Gamma$. If $f \in B$ we write $\tilde{f}$ for the corresponding function in $B_{G}$. We recall that for $f$ in $B$, we have associated to it a formal Dirichlet development (see [3]) which we write as

$$
f(w) \sim \sum_{x \geq 0} \hat{f}(x) e^{-x w}+\sum_{x<0} \hat{f}(x) e^{-|x| w} .
$$

The numbers $\hat{f}(x)$ are the Fourier coefficients of the restriction of $\tilde{f}$ to $\Gamma$.

(2.2) Theorem. There exists $f$ in $B$ such that $\Sigma_{x \geq 0} \hat{f}(x) e^{-x w}$ is not the Dirichlet series of a function in $A_{1}$.

Proof. Suppose that it is not the case; we have then that whenever $f$ is in $B$, it can be written as the sum of two functions, one in $A_{1}$ and another in $A_{1}^{0}$. As this decomposition is unique we have a linear isomorphism between $B$ and $A_{1} \times A_{1}^{0}$. On the other hand, all the spaces involved are Banach spaces with respect to uniform convergence; thus, we can make the cartesian product $A_{1} \times A_{1}^{0}$ into a Banach space by defining $\|(f, g)\|=\|f\|+\|g\|$. The open mapping theorem implies that there exists a constant $k$ such that

$$
\text { if } f=f_{1}+f_{1}^{0}\left(f_{1} \in A_{1}, f_{1}^{0} \in A_{1}^{0}\right) \text {, then }\left\|f_{1}\right\|+\left\|f_{1}^{0}\right\| \leq k\|f\| \text {. }
$$

Consider the linear functional $L$ on $B$ defined by $L(f)=f_{1}\left(2^{-1}\right)$; it is continuous because of (d). Consider now $1>r>0$ and the restriction of $f$ to $\{0 \leq \operatorname{Re} w \leq r\}$

$$
\left|f_{1}\left(2^{-1}\right)\right| \leq\left\|f_{1}\right\| \leq \sup \left\{\left|f_{1}(r w)\right|: \operatorname{Re} w \geq 0\right\}+\sup \left\{\left|f_{1}^{0}(r w)\right|: \operatorname{Re} w \leq 1\right\} .
$$

Calling $f_{1}^{r}(w)=f_{1}(r w)$ and $f_{1}^{0, r}(w)=f_{1}^{0}(r w)$ we get

$$
\left|f_{1}\left(2^{-1}\right)\right| \leq\left\|f_{1}^{r}\right\|+\left\|f_{1}^{0, r}\right\| \leq k\left\|f_{1}^{r}+f_{1}^{0, r}\right\|=k \sup \{|f(w)|: \operatorname{Re} w \leq r\} .
$$

Letting now $r$ tend to 0 we obtain ( $f$ is uniformly a.p. in the strip) 


$$
\left|f_{1}\left(2^{-1}\right)\right|=|L(f)| \leq k \sup \{|f(i y)|\}=k \sup \{|\tilde{f}(\alpha)|: \alpha \in \Gamma\} \text {. }
$$

This permits us to define a new continuous functional $L^{\prime}$ on the restriction of $B$ to $\Gamma$, and then extend it to a continuous functional on $C(\Gamma)$. It is clear that

$$
L^{\prime}(\hat{x})= \begin{cases}\left(e^{-1 / 2}\right)^{x} & \text { if } x \geq 0 \\ 0 & \text { if } x<0\end{cases}
$$

and this leads to a contradiction with (1.3).

\section{BIBLIOGRAPHY}

1. R. Arens and I. M. Singer, Generalized analytic functions, Trans. Amer. Math. Soc. 81 (1956), 379-393. MR 17,1226.

2. R. Arens, A Banach algebra generalization of conformal mappings of the disc, Trans. Amer. Math. Soc. 81 (1956), 501-513. MR 17,1226.

3. A. S. Besicovitch, Almost periodic functions, Cambridge Univ. Press, 1932.

4. E. Bishop, Representing measures for points in a uniform algebra, Bull. Amer. Math. Soc. 70 (1964), 121-122. MR 28 \#1510.

5. H. Bohr, Zur Theorie der fastperiodischen Funktionen. III. Dirichletentwicklung analytischer Funktionen, Acta Math. 47 (1926), 237-281.

6. J. Favard, Sur les fonctions harmoniques presque pêriodiques, Thèse, Paris, 1927, Gauthier-Villars, Edite urs.

7. T. W. Gamelin, Uniform algebras, Prentice-Hall, Englewood Cliffs, N. J., 1969.

8. A. M. Gleason, Function algebras, Seminars on Analytic Functions, vol. II, Institute for Advanced Study, Princeton, N. J., 1957, pp. 213-226.

9. K. Hoffman, Fatou's theorem for generalized analytic functions, Seminars on Analytic Functions, vol. II, Institute for Advanced Study, Princeton, N. J., 1957, pp. 227-239.

10. Boundary behavior of generalized analytic functions, Trans. Amer. Math. Soc. 87 (1958), 447-466. MR 20 \#3424.

11. K. Hoffman and I. M. Singer, Maximal subalgebras of $C(\Gamma)$, Amer. J. Math. 79 (1957), 295-305. MR 19,46.

12. K. Hoffman, Analytic functions and logmodular Banach algebras, Acta Math. 108.(1962), 271-317. MR $26 \# 6820$.

DEPARTAMENTO DE MATEMÁTICA, FACULTAD DE CIENCIAS EXACTAS Y NATURALES, CIUDAD UNIVERSITARIA, NÚNEEZ, BUENOS AIRES, ARGENTINA 